\title{
Application of Negative Pressure Wound Therapy with Instillation and Dwell Time of the Open Abdomen: Initial Experience
}

Luis G. Fernandez ${ }^{1}$, Pablo Sibaja Alvarez ${ }^{2}$, Mark J. Kaplan ${ }^{3}$, Alfredo A. Sanchez-Betancourt ${ }^{4}$, Marc R. Matthews ${ }^{5}$, Alan Cook 6

1. Surgery, Trauma Wound Care, University of Texas Health Science Center, Tyler, USA 2. General Surgery, Universidad Federada San Judas Tadeo, San Jose, CRI 3. Surgery, Einstein Medical Center, Philadelphia, USA 4. Medical Administration, Hospital Mexico, San Jose, CRI 5. Surgery, Arizona Burn Center, Phoenix, USA 6. Epidemiology and Biostatistics, University of Texas Health Science Center, UT Health East Texas, Tyler, USA

Corresponding author: Luis G. Fernandez, thebigkahuna115@gmail.com

\begin{abstract}
Recent therapeutic advances in the management of severe abdominal sepsis (SAS) have improved patient mortality and morbidity. However, SAS and its impact on multiple organ failure remain a serious, lifethreatening condition with a high mortality rate. The open abdomen (OA) technique has become an effective alternative to repeat laparotomy. The use of OA negative pressure wound therapy (OA NPWT) has been a significant advancement in the management of the open abdomen. Similarly, negative pressure wound therapy (NPWT) with instillation and dwell time (NPWT-i) has been used in patients with multiple comorbidities, with an American Society of Anesthesiology Classification $\geqslant 2$, severe traumatic wounds, diabetic foot infections, and wounds complicated by invasive infection or extensive biofilm. Controlled instillation of saline during NPWT-i may further enhance healing by facilitating automatic and contained volumetric wound irrigation and cleansing and diluting local levels of inflammatory cytokines, improving the local as well as the systemic response to infection. Although the soft tissue and intra-abdominal compartments differ anatomically, they share very similar biologic responses to infections. Therefore, from a biologic and physiologic aspect, intraperitoneal instillation therapy may play a role as an adjunctive treatment of abdominal compartment inflammation from trauma or infection. The addition of saline solution instillation to OA NPWT (OAI) in a programmed, controlled manner may offer the clinician an effective adjunctive therapy for the treatment of the complex septic abdomen. The technical aspects of instillation into the OA and a pooled multicenter case study cohort utilizing OAI with saline solution, bacitracin, or hypochlorous acid in the management of the septic abdomen is presented.
\end{abstract}

Categories: Emergency Medicine, General Surgery, Trauma

Keywords: open abdomen, negative pressure wound therapy, wound healing, abdominal cavity, peritoneal lavage

\section{Introduction}

The open abdomen $(\mathrm{OA})$ technique using temporary abdominal closure (TAC) has been shown to be beneficial in the care of patients with complex abdominal pathology [1-3]. The TAC approach allows for a step-by-step approach to address the underlying pathologic process, offering patients the opportunity to recover from the initial shock state and achieve a level of physiologic homeostasis and allowing further surgical repair in a more controlled environment.

Early methods of TAC were passive and provided coverage for the abdominal contents; however, these techniques failed to adequately address intra-abdominal fluid/lymph and ongoing inflammation. The application of OA negative pressure wound therapy (OA NPWT) as a form of TAC (OA NPWT, Abthera ${ }^{\mathrm{TM}}$ Open Abdomen Negative Pressure Therapy, KCI, an Acelity Company, San Antonio, TX, US) has provided the surgeon with a more technically advanced device to assist in the care of patients with a complex abdominal pathology [4-5]. With the ability to remove intraperitoneal fluid while limiting lateral abdominal wall retraction (and in many cases, aiding in medial abdominal wall closure), these innovative forms of TAC represent dynamic systems that have improved overall patient outcomes and have become the preferred mode of TAC in patients [6].

Abdominal washouts (direct fluid irrigation into the peritoneal cavity) have been an integral part of the total management of the OA and has been used to help remove bacteria and other inflammatory mediators. However, washouts typically require a trip to the operating room (OR), which may not be feasible in some critically ill OA patients. Therefore, having a contained instillation system may provide a better option. Adding the instillation of a biocompatible solution to negative pressure wound therapy (NPWT-i), in a controlled manner, may offer the clinician an additional tool for the management of the complex septic abdomen.

We describe an easy-to-use method of intermittent fluid instillation within the open abdomen that 


\section{Cureus}

effectively distributes fluid throughout the peritoneal cavity and the visceral contents. This technique incorporates standard negative pressure therapy and an additional set of tubing to allow for the flow of fluids. TAC with intra-abdominal fluid instillation may offer several advantages, over other modalities of TAC with repeated multiple laparotomies, in the management of the open abdomen.

\section{Materials And Methods \\ Study design}

This retrospective small, pooled case series, included seven patients with severe abdominal sepsis. OA instillation (OAI) was utilized in the treatment of all patients. The type of fluid instilled (saline solution, bacitracin, or hypochlorous acid) in each patient was selected by the treating physician.

During the intraoperative assessment, each abdomen was classified according to the amended OA classification system (Table 1) [7]. Duodenal and liver injury were graded according to the American Association of Surgery for Trauma (AAST) Organ Injury Scale (Table 2 and Table 3, respectively) [8]. The Hinchey scale for perforated diverticulitis was also used (Table 4) [9].

\begin{tabular}{|ll|}
\hline Grade & Description \\
\hline 1A & Clean open abdomen without adherence between the bowel and abdominal wall or fixity \\
1B & Contaminated open abdomen without adherence between the bowel and abdominal wall or fixity \\
1 C & Enteric leak without fixation \\
2A & Clean open abdomen developing adherence between the bowel and fixity \\
2B & Contaminated open abdomen developing adherence between the bowel and fixity \\
2C & Enteric leak developing fixation \\
\hline 3A & Clean, frozen abdomen \\
3B & Contaminated, frozen abdomen \\
4 & Established enteroatmospheric fistula, frozen abdomen \\
\hline TABLE 1: Classification of the open abdomen \\
Adapted from Björck et al. [7] \\
\hline \hline
\end{tabular}




\section{Cureus}

\begin{tabular}{|l|l|l|}
\hline Grade & Damage & Injury Description \\
\hline II & Hematoma & Single portion of the duodenum \\
& Laceration & Partial-thickness injury without perforation \\
Hematoma & More than one portion \\
III & Laceration & Disruption $<50 \%$ of circumference \\
IV & Laceration & Disruption $50-75 \%$ of the circumference of the second portion \\
V & Disruption of $50-100 \%$ of the circumference of the first, third, and fourth portion \\
& Laceration & Disruption $>75 \%$ of the circumference of the second portion \\
& Involving ampulla or distal common bile duct \\
& Mascular & Devascularization of duodenum
\end{tabular}

\section{TABLE 2: Duodenum injury scale}

Adapted from Tinkoff et al. [8]

\begin{tabular}{|c|c|c|}
\hline Grade & Damage & Injury Description \\
\hline \multirow[t]{2}{*}{ I } & Hematoma & Subcapsular, non-expansive, $<10 \%$ of surface \\
\hline & Laceration & Non-bleeding, $<1 \mathrm{~cm}$ deep \\
\hline \multirow[t]{2}{*}{ II } & Hematoma & Subcapsular, non-expansive, $10-50 \%$ of surface \\
\hline & Laceration & $1-3 \mathrm{~cm}$ deep, $<10 \mathrm{~cm}$ in size \\
\hline \multirow[t]{2}{*}{ III } & Hematoma & Subcapsular, expansive $>50 \%$ of surface or intraparenchymal $>2 \mathrm{~cm}$ \\
\hline & Laceration & $>3 \mathrm{~cm}$ deep \\
\hline \multirow[t]{2}{*}{ IV } & Hematoma & Bleeding intraparenchymal rupture \\
\hline & Laceration & Involving $25 \%-50 \%$ of lobe \\
\hline \multirow[t]{2}{*}{ V } & Laceration & Parenchymal, involving more than $50 \%$ of lobe \\
\hline & Vascular & Juxtahepatic vein, main hepatic veins, or retrohepatic cava \\
\hline VI & Vascular & Hepatic avulsion \\
\hline \multicolumn{3}{|c|}{ TABLE 3: Liver injury scale } \\
\hline \multicolumn{3}{|c|}{ Adapted from Tinkoff et al. [8] } \\
\hline
\end{tabular}




\section{Cureus}

\begin{tabular}{|l|l|}
\hline Grade & Description \\
\hline I & Pericolic abscess \\
II & Pelvic, intraabdominal, or retroperitoneal abscess \\
III & Generalized purulent peritonitis \\
IV & Generalized fecal peritonitis \\
\hline
\end{tabular}

\section{TABLE 4: Perforated diverticulitis scale}

Adapted from Hinchey et al. [9]

\section{Damage control surgery}

Damage control surgery was performed on all patients following principles, as originally described by Rotondo et al., for the resuscitation of trauma patients [10]. The component steps of DCS are as follows:

- Part zero (DC 0): Early/rapid, severe injury pattern recognition for potential damage control candidates.

- Part one (DC I) Immediate exploratory celiotomy with rapid control of bleeding, contamination, abdominal packing, and temporary wound closure in the operating room.

- Part two (DC II): Controlled resuscitative phase for physiological and biochemical stabilization) and a thorough tertiary examination to identify all injuries in the intensive care unit (ICU).

- Part three (DC III): Re-exploration to perform definitive repair of all injuries, occurring once physiology has normalized.

\section{Open abdomen instillation}

OAI is initiated in the operating room (or, if needed, at the patient's bedside, in the surgical intensive care unit (ICU)). Application of OAI has been previously described [11]. Briefly, following abdominal washout, the OA fenestrated visceral protective layer (Abthera ${ }^{\mathrm{TM}}$ Fenestrated Visceral Protective Layer, KCI, an Acelity Company) is cut to size (as needed), placed over the abdominal contents, and the edges tucked into the paracolic gutters (Figures $1 \mathrm{~A}-1 \mathrm{C}$ ). The perforated foam dressing $\left(\mathrm{Abthera}{ }^{\mathrm{TM}}\right.$ ) is then placed directly on top of the visceral protective layer followed by the application of drape (Abthera ${ }^{\mathrm{TM}}$ ) ensuring an overlap of at least $8 \mathrm{~cm}$ over the periwound skin (Figure $1 D$ ). A dual lumen port (V.A.C. VeraT.R.A.C. ${ }^{\text {TM }}$ Pad, KCI) or double port tubing set (V.A.C. VeraT.R.A.C. DUO ${ }^{\mathrm{TM}}$ ) is then placed over the occlusive foam dressing for the delivery of negative pressure and the instillation of a biocompatible solution (Figures $1 E-1 F$ ). The double port tubing set contains two pads for larger or vertical wounds that provide for the individualized delivery of negative pressure and the instillation solution. This allows for customized pad placement to enhance fluid circulation and removal. The tubing set is connected to the fully automated NPWT-i unit (V.A.C. Ulta ${ }^{\mathrm{TM}}{ }^{\mathrm{T}}$ Therapy unit, $\mathrm{KCI}$ ) to provide both controlled instillation and aspiration of the $\mathrm{OA}$ instillation solution. 


\section{Cureus}
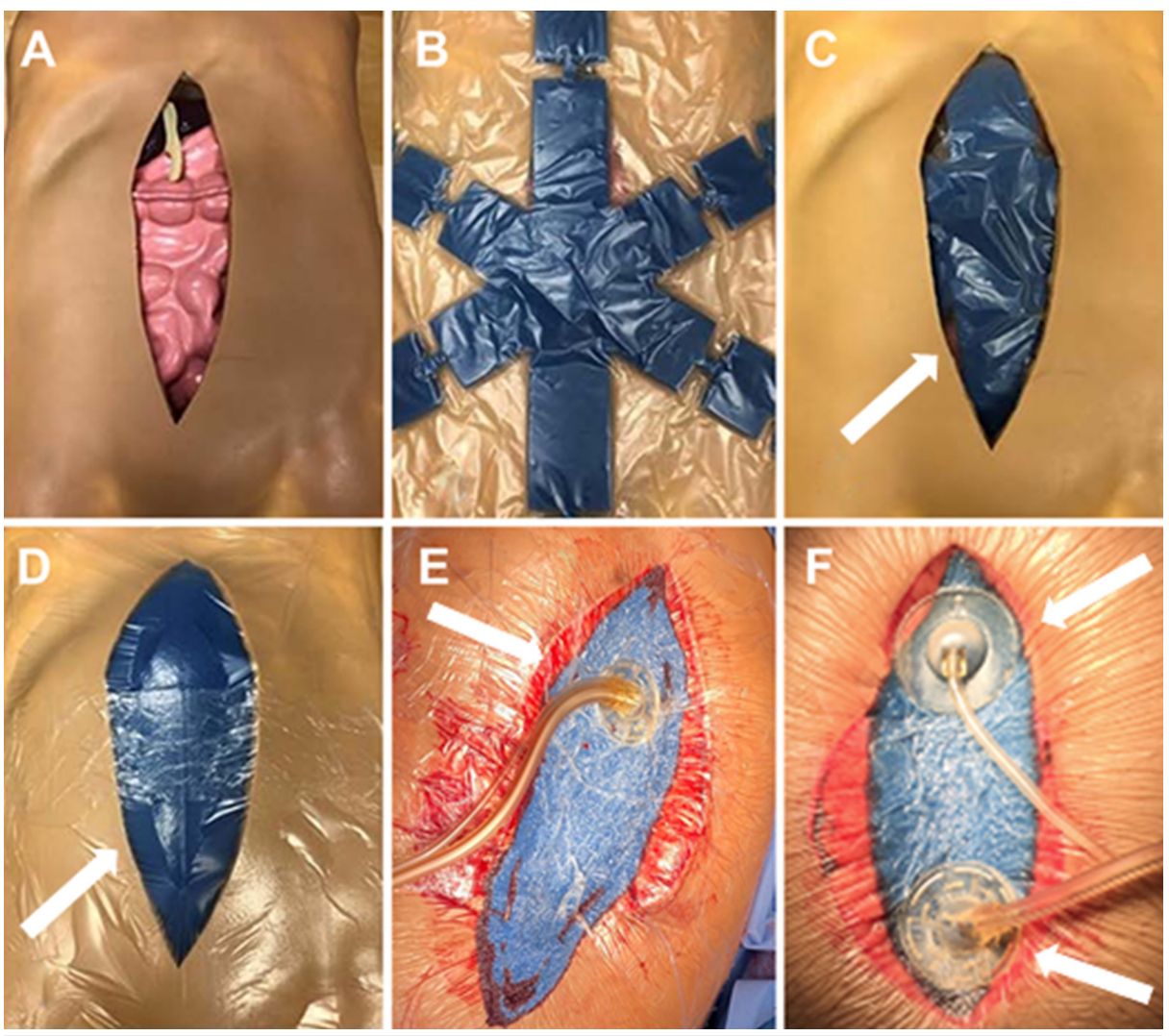

\section{FIGURE 1: Application of open abdomen instillation}

A. Open abdomen; B. Visceral protective layer; C. Visceral protective layer placed over the abdominal contents in the abdominal cavity; D. Intraabdominal placement of perforated foam dressing; E. Placement of dual lumen port tube set; F. Placement of the double port tube set.

Adapted from Matthews et al. [11]

An additional fluid container of one liter of the instillation solution should be connected and replaced, as needed, depending on the treatment parameters. The holding canisters should also be replaced as often as required to guarantee that the $\mathrm{OA}$ instillation can be properly delivered. Suggested treatment parameters are described in Table 5.

\begin{tabular}{|l|l|}
\hline Category & Suggested Parameter \\
\hline Pressure setting & $100-125 \mathrm{mmHg}$ \\
Instillation volume & $200-300 \mathrm{~mL}$ (per cycle) \\
Dwell time & $20-30$ minutes \\
Negative pressure cycle time & 120 minutes \\
Total treatment cycle time & $140-160$ minutes \\
Total cycles per day & 10 \\
\hline
\end{tabular}

\section{TABLE 5: Suggested treatment parameters}

\section{Results}

\section{Patient 1: irrigation of a grade 3B open abdomen}

A 57-year-old, morbidly obese male presented to the emergency department for the evaluation of acute abdominal pain, with a $10-\mathrm{cm}$ area of induration, erythema, tenderness, and swelling of the midline of the 
abdomen (Figure $2 \mathrm{~A}$ ). The patient had a history of exploratory laparotomy for a perforated duodenal ulcer within the last 10 months with a Graham Patch repair. Laboratory blood tests revealed elevated white blood cell (WBC) counts. An abdominal computed tomography (CT) scan with intravenous (IV) contrast was performed and indicated a possible bowel perforation with anterior abdominal wall abscess (Figure $2 B$ ). The patient was taken to surgery. Operative findings included a mesogastric incisional hernia containing a portion of the transverse colon, a deep right upper quadrant abscess tract, a small amount of enteric fluid, and frank purulent discharge. A limited exploratory celiotomy and debridement of the ventral hernia abscess were performed. A 7-mm closed suction drain was placed in the right upper quadrant directed towards the suspected duodenal fistula. Open abdominal irrigation was initiated using $1 \mathrm{~L}$ of hypochlorous acid wound cleansing solution (Vashe ${ }^{\circledR}$, SteadMed ${ }^{\circledR}$, Fort Worth, TX, US; off-label usage) with 10-minute dwell time. After the initial irrigation, a non-adhering silicone dressing (Adaptic Touch ${ }^{\mathrm{TM}}$ Non-Adhering Silicone Dressing, Systagenix, an Acelity Company, Gargrave, UK) was applied, and OAI initiated (instilling 200 cc normal saline with 20-minute dwell time, followed by two hours of negative pressure at $-125 \mathrm{mmHg}$ ). The patient was admitted to the surgical/trauma unit and underwent intensive resuscitation. Upper gastrointestinal endoscopy revealed a perforated duodenal ulcer, and a covered stent was placed by the gastroenterologist (Figure 2C). Four days later, a follow-up CT scan revealed a small amount of residual air in the area of duodenal perforation; no abscess or bowel obstruction was noted. Prior to definitive closure, the patient underwent upper gastrointestinal fluoroscopic assessment, which showed no extravasation of contrast. The stent, which had been placed four days prior, had passed into the right colon (Figures $2 D-2 E$ ). The patient was taken for definitive abdominal wall closure on hospital day (HD) six. Re-exploration of the abdominal cavity was performed with a 360-degree myocutaneous flap advancement. The abdomen underwent reconstruction using a 10 × $20 \mathrm{~cm}$ reconstructive tissue matrix (Strattice ${ }^{\mathrm{TM}}$ Reconstructive Tissue Matrix, LifeCell, an Allergan affiliate, Branchburg, NJ, US), utilizing an overlay technique followed by direct, manual irrigation of the incision using a hypochlorous acid wound cleansing solution (Figures $2 F-2 H$ ). Bilateral subcutaneous drains were placed followed by skin closure using staples and the application of closed incision negative pressure therapy (ciNPT, Prevena ${ }^{\mathrm{TM}}$ Incision Management System, KCI, an Acelity Company, San Antonio, TX, US) (Figure 21). The patient progressively improved and was discharged home on HD 12. At the postoperative follow-up (76 days post-discharge), the patient was asymptomatic and living a normal life (Figures $2 J-2 K$ ).
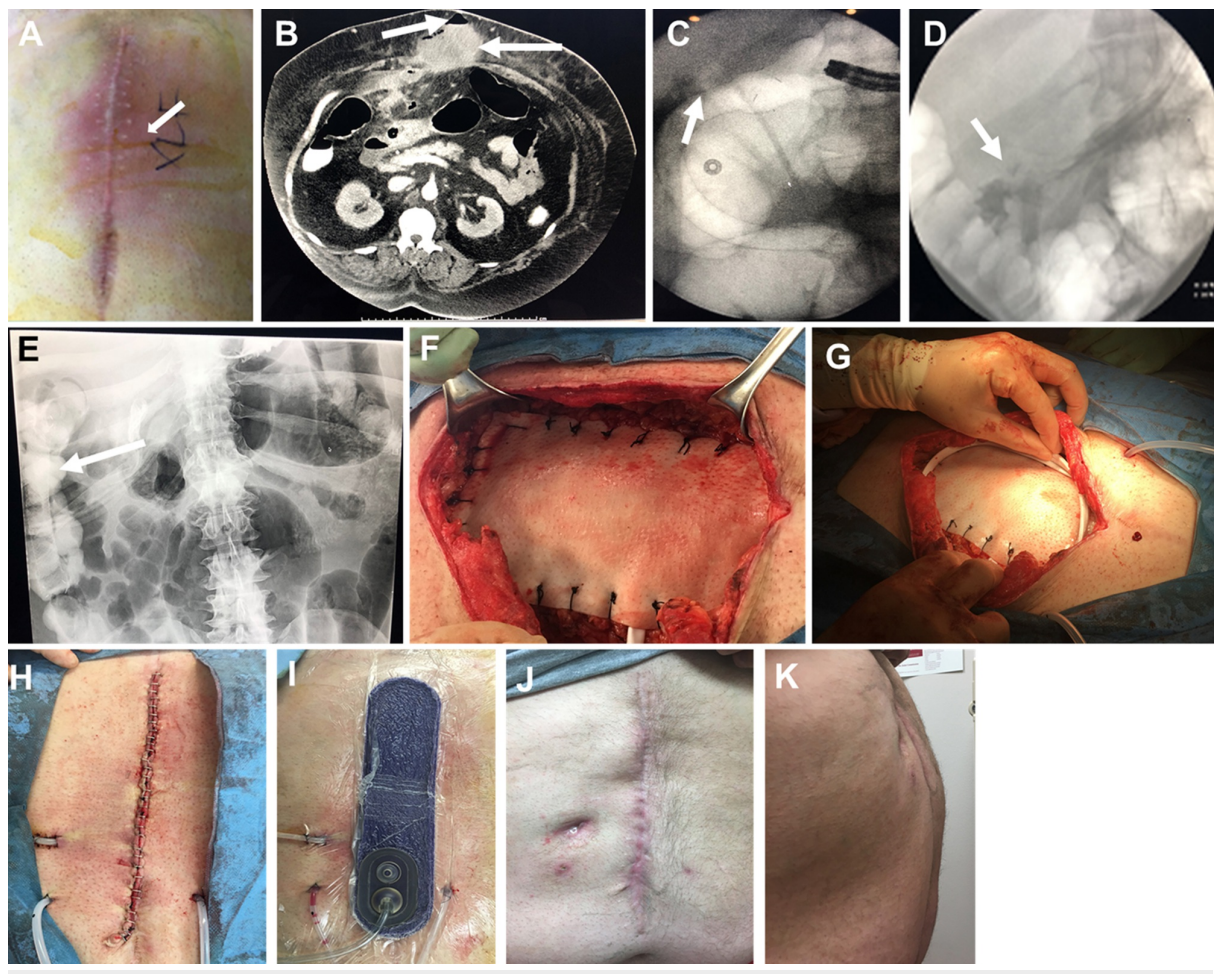

FIGURE 2: Open abdomen repair and instillation of a contaminated, frozen abdomen

A. Midline erythema; B. Abdominal CT showing potential bowel perforation (left arrow) and abdominal abscess (right arrow); C. Endoscopic duodenal stent placement; D. Elimination of duodenal leak achieved (arrow); E. Duodenal stent in right colon (arrow); F. Application of reconstruction tissue matrix; G. Drain application; H. Closed abdominal incision, I. Application of closed incision negative pressure therapy; J. Anterior view of fully healed abdominal incision; K. Lateral view of fully healed abdominal incision

Patient 2: perforated appendicitis with delayed presentation and septic 
abdomen, grade 2B open abdomen

A 76-year-old female with dementia and acute encephalopathy was admitted to the hospital. On physical examination, the patient displayed diffuse abdominal pain and diffuse peritonitis. A CT scan of the abdomen with oral contrast revealed a large pneumoperitoneum and diffuse fluid within the abdominal cavity, consistent with a perforated viscus (Figures 3A-3C). The patient underwent exploratory laparotomy, adhesiolysis, aerobic/anaerobic cultures, drainage or debridement of a pelvic/inter-loop abscess, and abdominal irrigation with $2 \mathrm{~L}$ of hypochlorous acid wound solution (off-label usage) with 10-minute dwell time. A fibrin sealant (Tisseel, Baxter International Inc., Deerfield, IL, US) in the right upper and lower quadrants was added, followed by the placement of a mechanical bioresorbable adhesion barrier (Seprafilm, Sanofi-Aventis, Bridgewater, NJ, US) and initiation of NPWT-i (instillation of 200 cc normal saline with 20minutes dwell time, followed by negative pressure therapy ( $-125 \mathrm{mmHg}$ ) for two hours). Intraoperative findings were consistent with perforated appendicitis with delayed presentation and a septic abdomen, Grade 2B (Figures 3D-3E). The patient was admitted to the intensive care unit for continued aggressive resuscitation. She was taken for abdominal washout and closure 48 hours after the index operation. The operating surgeon described no purulent fluid or fibrin deposition and minimal adhesions. There was evidence of an inflammatory reaction in the right lower quadrant, with normal-appearing large and small bowel. The patient underwent primary closure and continued to improve. The patient was discharged to a skilled nursing facility.
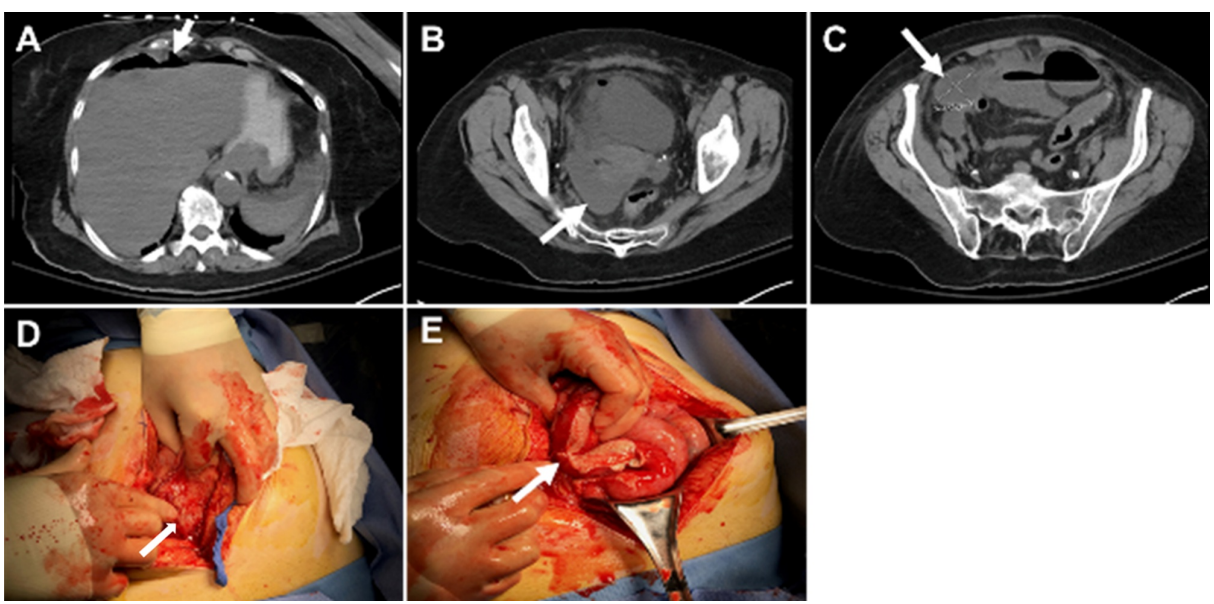

FIGURE 3: Perforated appendicitis with delayed presentation and septic abdomen, grade 2B open abdomen

A. Pneumoperitoneum identified in the patient's abdomen (arrow); B. Diffuse peritoneal fluid identified in abdominal CT scan (arrow); C. Diffuse peritoneal fluid identified in abdominal CT scan (arrow); D. Diffuse inflammation of bowel (arrow); E. Fibrin deposition involving the large and small bowel (arrow)

\section{Patient 3: blunt traumatic grade III injury to the second portion of the duodenum and grade III blunt liver laceration septic abdomen, grade 2B open abdomen}

A 20-year-old female presented to the emergency department (ED) with back pain that began earlier when the patient was thrown off a horse and the horse landed on her. The pain was located primarily in the cervical and thoracic sections of her back. Associated symptoms include right-sided pelvic pain, abdominal pain, and rib pain. The abdominal pain was severe and was located in the upper and lower sections of her abdomen. Figure $4 \mathrm{~A}$ illustrates the saddle pommel imprint on the patient's right upper quadrant. A CT of the abdomen and pelvis with IV contrast was obtained and showed fluid around the duodenum, suggesting a possible duodenal rupture (Figure $4 B$ ). The patient was taken emergently for exploratory laparotomy. The patient underwent surgical exploration via a midline incision, a right Cattel -Brasch medial rotation with a Kocker maneuver, exploration of the lesser sac, debridement, and two-layer repair of the second portion of duodenum with Graham Patch placement, repair of the Grade III left lobe of liver laceration with the argon beam coagulator and round/falciform ligament patch, abdominal washout, placement of a fibrin sealant on the duodenal/liver repair (Figures $4 \mathrm{C}-4 \mathrm{~F}$ ), and placement of $\mathrm{OA}$ instillation and support lines. Instillation was initiated once the patient was in the surgical intensive care unit. Therapy parameters include the instillation of $200 \mathrm{cc}$ normal saline with a dwell time of 20 minutes followed by negative pressure (-125 $\mathrm{mmHg}$ ) for two hours. Before definitive closure, a CT of the abdomen and pelvis, as well as a magnetic resonance imaging (MRI) scan of the biliary system, was performed. No surgical lesions were identified (Figures $4 G-4 H$ ). 


\section{Cureus}

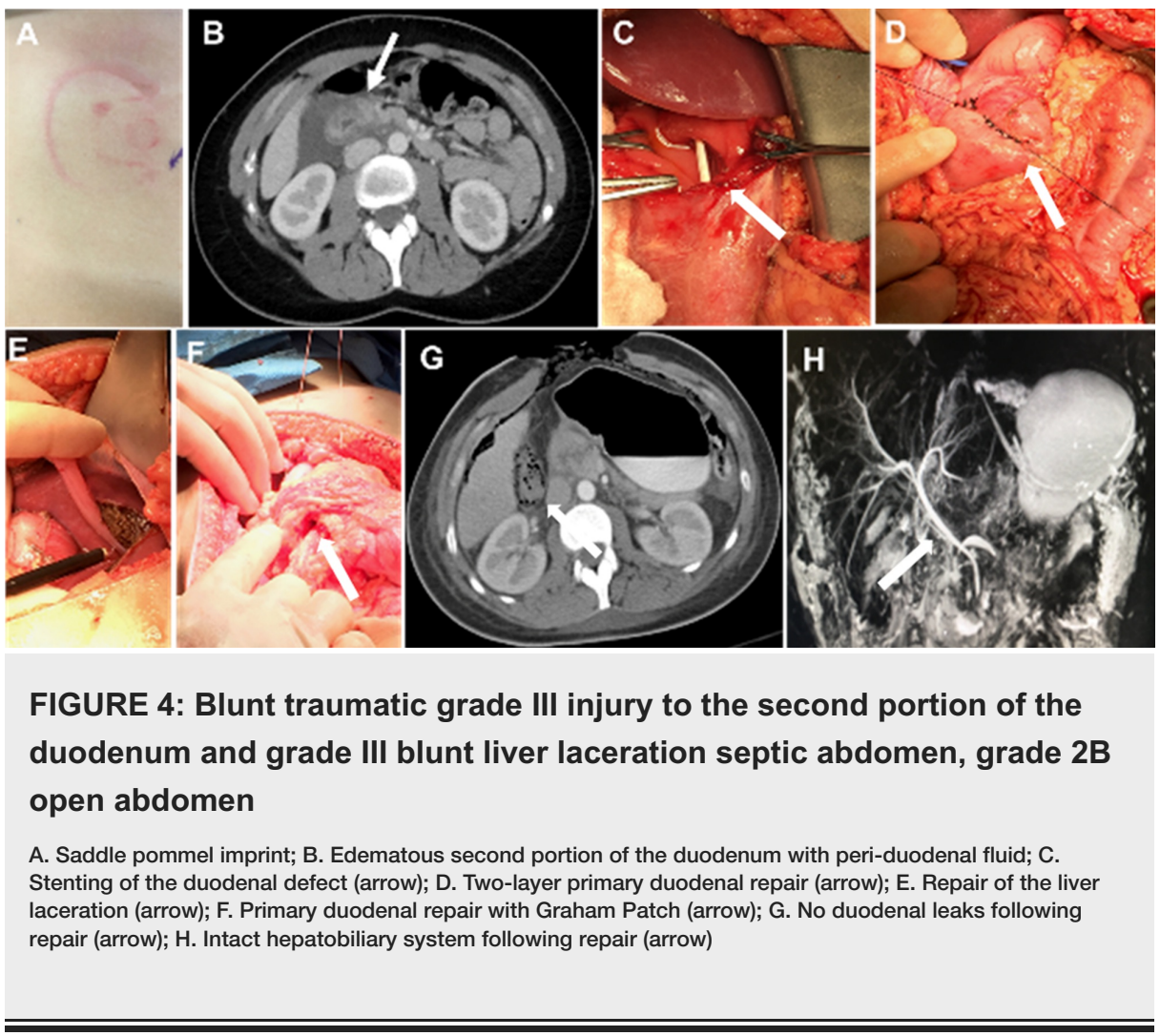

On postoperative day three, the patient underwent exploratory laparotomy, abdominal washout, placement of a distal feeding jejunostomy tube, closed suction drain placement along the right upper quadrant, and primary closure of the abdominal wall. No evidence of a duodenal leak or significant bowel

edema/inflammation was noted intraoperatively. Closed incision negative pressure therapy was applied over the closed incision and left in place for six days. Prior to the closure of the incision, bupivacaine was injected into the fascia and subcutaneous tissue to help manage postoperative pain. A fluoroscopic upper gastrointestinal contrast study was obtained on postoperative day six. No evidence of a leak from the duodenal repair was observed. The patient progressively improved, tolerating a general diet and ambulation, and was discharged home on HD 13.

\section{Patient 4: gunshot wound to the abdomen (grade II injury)}

A 26-year-old male presented with a gunshot wound to the abdomen. AAST Grade 2 sigmoid and colon injuries were observed during the abdominal assessment. The sigmoid and colon injuries were repaired, and the abdomen left open for 24 hours, utilizing the OA NPWT System as a TAC, followed by primary closure.

Ten days after surgery, the patient developed intra-abdominal sepsis secondary to an anastomotic breakdown with antibiotic-resistant Escherichia coli. Systemic antibiotics were initiated. The patient's abdomen could not be closed secondary to bowel edema and infection (Figure 5A). Re-exploration surgery was performed, and OAI initiated. Normal saline $(500 \mathrm{cc})$ and bacitracin $(1 \mathrm{~g})$ were instilled into the OA, followed by a 15 -minute dwell time and four hours of continuous negative pressure $(-125 \mathrm{mmHg}$ ) (Figure $5 B$ ). The patient underwent re-exploration surgery every 48 hours to drain the loculated pus and undergo dressing changes. After 10 days, the infection had resolved; however, the abdomen was unable to be closed, and a dermal regeneration template (Integra ${ }^{\circledR}$ Dermal Regeneration Template, Integra LifeSciences, Plainsboro, NJ, US) was placed over the peritoneal contents to stimulate the development of granulation tissue (off-label usage) (Figure 5C). After healthy granulation tissue covered the wound bed, the patient underwent a split-thickness skin graft to the abdominal wall. Traditional NPWT (V.A.C. ${ }^{\circledR}$ Therapy, KCI) was used over the graft and left in place for five days (Figure $5 D$ ). The patient was discharged from the hospital and is awaiting definitive abdominal wall closure. 


\section{Cureus}
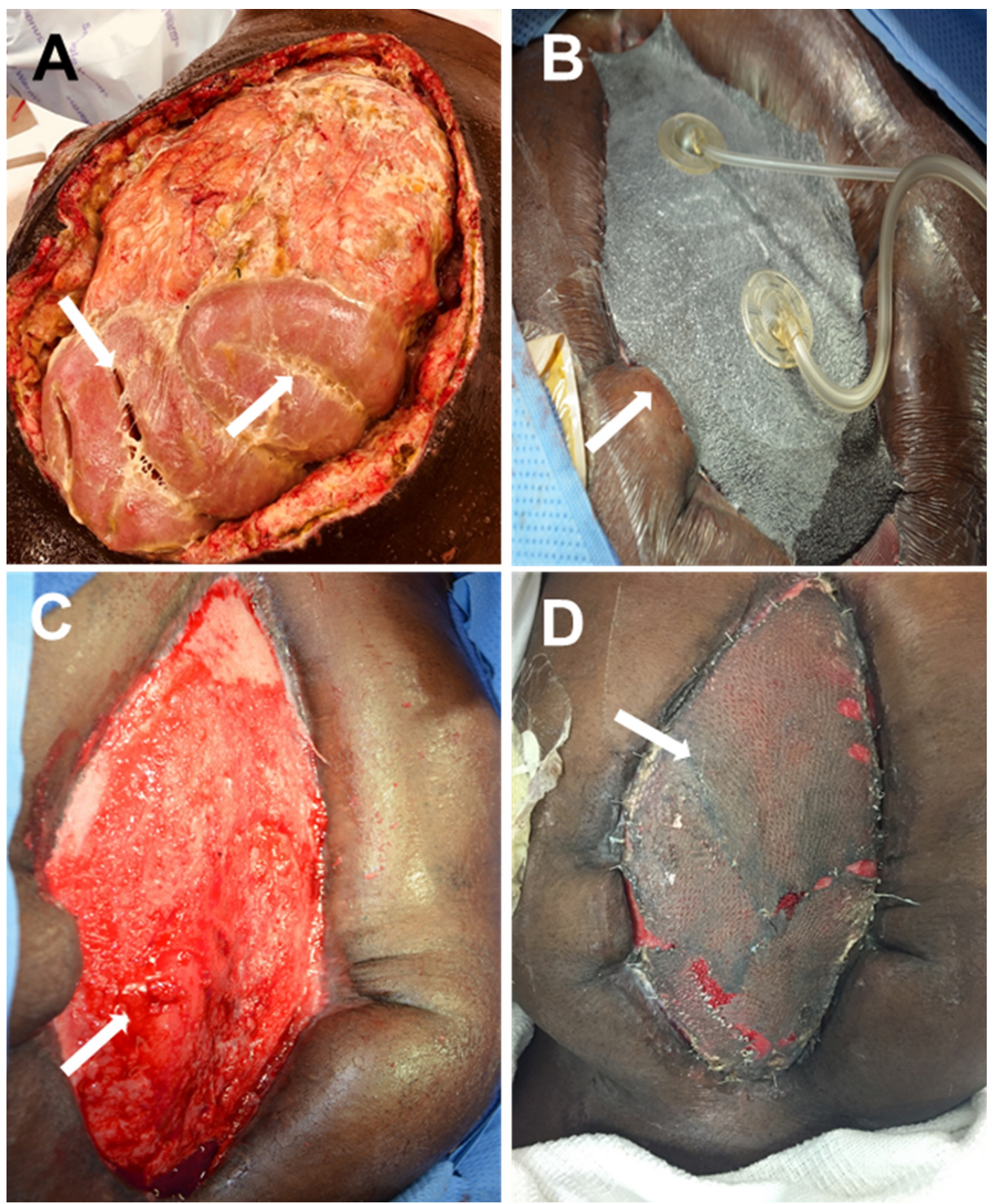

FIGURE 5: Gunshot wound to abdomen with Grade 2 injury to sigmoid and colon

A. Intraoperative exudate and pockets of gross purulence; B. Application of open abdomen instillation; C. Granulation tissue developing over the dermal regeneration template; D. Abdomen five days after NPWT use over split-thickness skin graft

\section{Patient 5: grade III perforated colon and intra-abdominal sepsis}

A 65-year-old female presented with a perforated colon and intra-abdominal sepsis (Hinchey Grade III). Systemic antibiotics were given at presentation. Damage control surgery was performed with resection of the sigmoid colon. OAI was initiated at the time of the index surgery, with $500 \mathrm{cc}$ normal saline and $1 \mathrm{~g}$ of bacitracin with a dwell time of 15 minutes, followed by four hours of continuous negative pressure (-125 $\mathrm{mmHg}$ ). After five days of OAI, sepsis was controlled (Figure 6A). Due to massive bowel edema, the abdomen could not be closed, and a reconstructive tissue matrix was applied (Figure 6B). Instillation using NPWT-i on the abdominal wall wound was initiated (using 500 cc normal saline, 15-minute dwell time, and four hours of continuous negative pressure) to prevent desiccation and promote the formation of granulation tissue.

Granulation tissue development was observed after 14 days of NPWT-i therapy (Figure 6C). A split-thickness skin graft was performed once the wound showed $100 \%$ coverage of granulation tissue (Figure $6 D$ ). Traditional NPWT was placed on the graft as a bolster for five days. 


\section{Cureus}
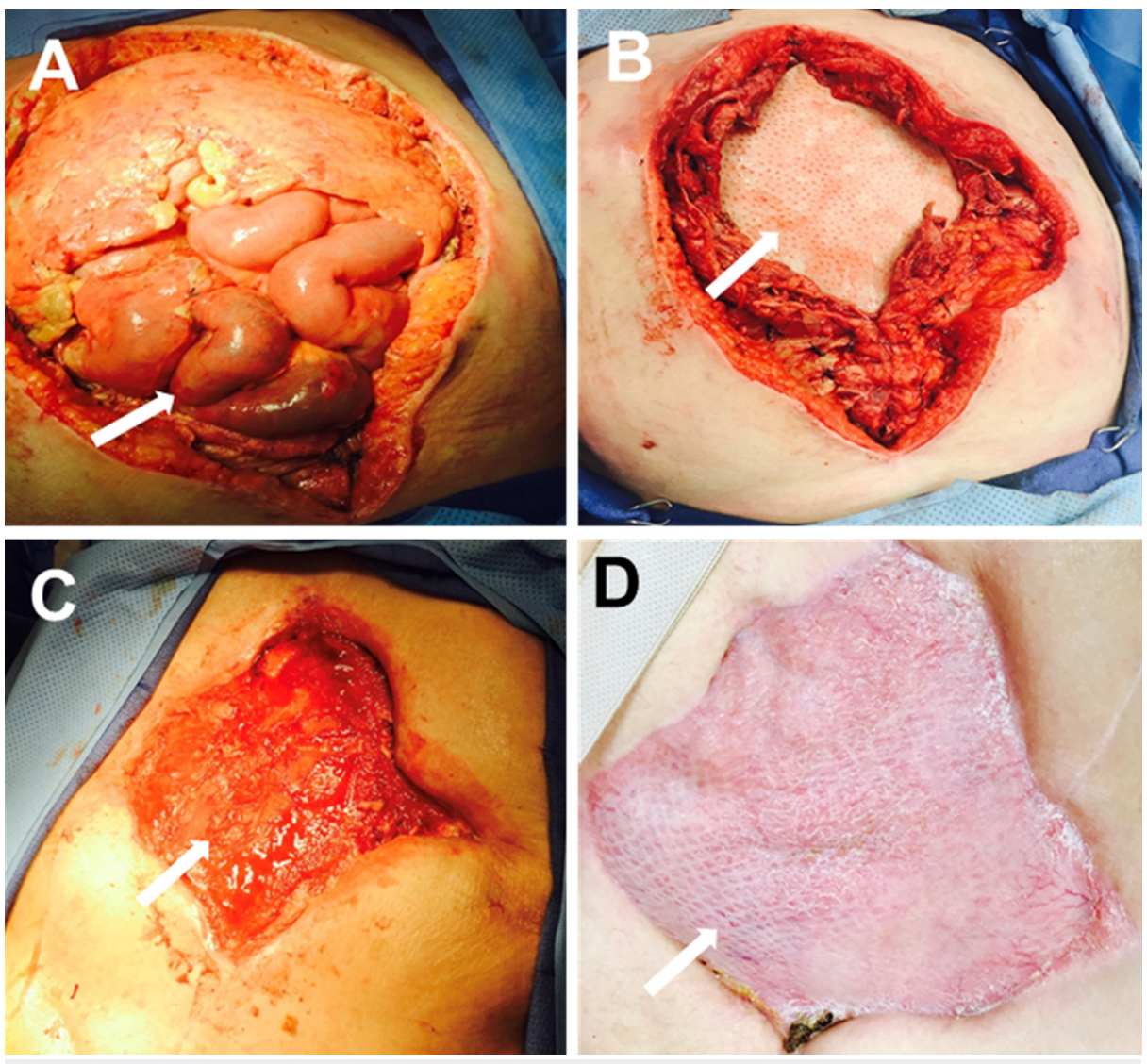

FIGURE 6: Perforated colon and intra-abdominal sepsis

A. Open abdomen after five days of instillation therapy; B. Reconstructive tissue matrix placement; C. Granulation tissue development after two weeks of instillation; D. Application of split-thickness skin graft

\section{Patient 6: grade III perforated diverticulitis and diffuse peritonitis}

A 32-year-old morbidly obese male presented to the ED for evaluation due to a five-day lower abdominal pain, distension, and fever. Patient assessment showed signs of peritoneal irritation with a positive McBurney sign and tenderness of the pelvic region. Abdominal ultrasound reported cholecystolithiasis with a non-inflamed gall bladder and findings compatible with acute appendicitis without specific fluid collection (Figures 7A-7B). A Mannheim Peritonitis Index Score of 16 was calculated [12]. The patient underwent exploratory surgery. Active diffuse suppurative peritonitis with adhesions and multiple contaminated peritoneal fluid collections (OA Grade 2B) were observed (Figure 7C). Fluid samples were obtained for cultures. Extensive saline lavage and debridement were performed. A perforated sigmoid diverticulitis and inflamed sigmoid colon (Hinchey Grade III) was identified. A resection of the sigmoid and a temporary colostomy (Hartman's' Procedure) were performed [13]. 


\section{Cureus}
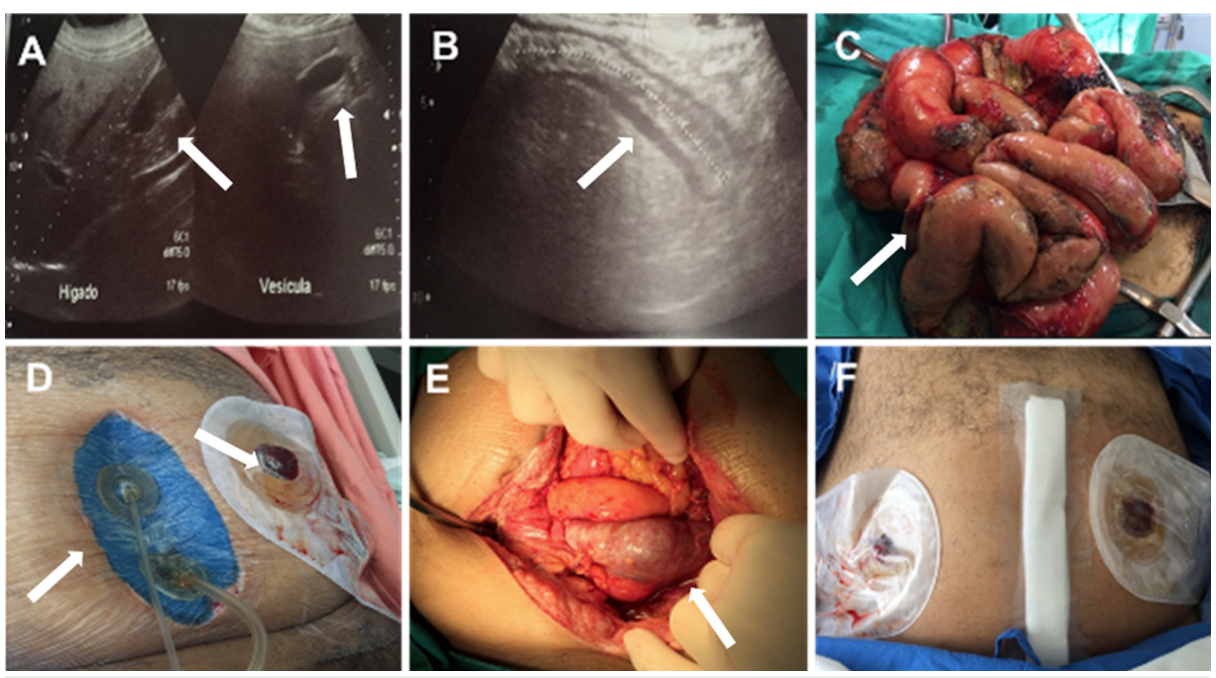

\section{FIGURE 7: Perforated diverticulitis and diffuse peritonitis}

A. Abdominal ultrasound showing cholecystolithiasis (arrows); B. Abdominal ultrasound showing acute appendicitis (arrow); C. Active diffuse suppurative peritonitis; D. Abdominal installation and temporary colostomy; E. Open abdomen 72 hours after abdominal instillation (arrow); F. Closed abdomen

Due to visceral edema, extensive contamination, and active peritoneal inflammation, a staged management treatment plan was initiated. OAI was initiated with $200 \mathrm{cc}$ saline instillation followed by a 20 -minute dwell time and two hours of continuous negative pressure at $-125 \mathrm{mmHg}$ (Figure $7 D$ ). Intravenous antibiotics, prophylactic anticoagulant medication, and analgesics were also given. At postoperative follow-up assessments, the patient showed reduced pain, increased appetite, decreased abdominal distention, and normalized vital signs. After 72 hours, the patient returned to the OR for abdominal exploration and surgical washout. The abdominal cavity was clean with reduced edema (Figure $7 E$ ), allowing for primary fascial closure in a non-tension environment with Penrose drain placement on the opposite pelvic side (Figure $7 F$ ). The abdominal closure procedure was performed without complications. Seven days after closure, the patient was discharged with no signs of active infection and oral tolerance to fluids and soft food.

\section{Patient 7: perforated appendicitis with delayed presentation and septic abdomen (grade 2B)}

A 32-year-old male with no history of chronic disease presented to the ED for the second time in a five-day period, complaining of abdominal pain present for 10 days, oral intolerance, vomiting, and multiple foulsmelling liquid stools. Patient evaluation showed signs of dehydration, tachycardia, fever, and abdominal distension with abdominal rebound tenderness and induration of the right lower quadrant. An abdominal ultrasound showed concentrated non-mobile edematous small bowel and cecum aggregation in the right lower quadrant wrapping around a fluid collection with a calculated volume of $56 \mathrm{cc}$ consistent with a retrocecal pelvic abscess displacing the bladder and rectum (Figures $8 A-8 B$ ). A portion of a tubular thickened structured, possibly a portion of the bowel, adjacent to the fluid collection was described. The patient was diagnosed with a perforated retrocecal appendicitis with an interloop pelvic abscess and potential mechanical intestinal obstruction. The patient had a Mannheim Peritonitis Index Score of 21. 


\section{Cureus}
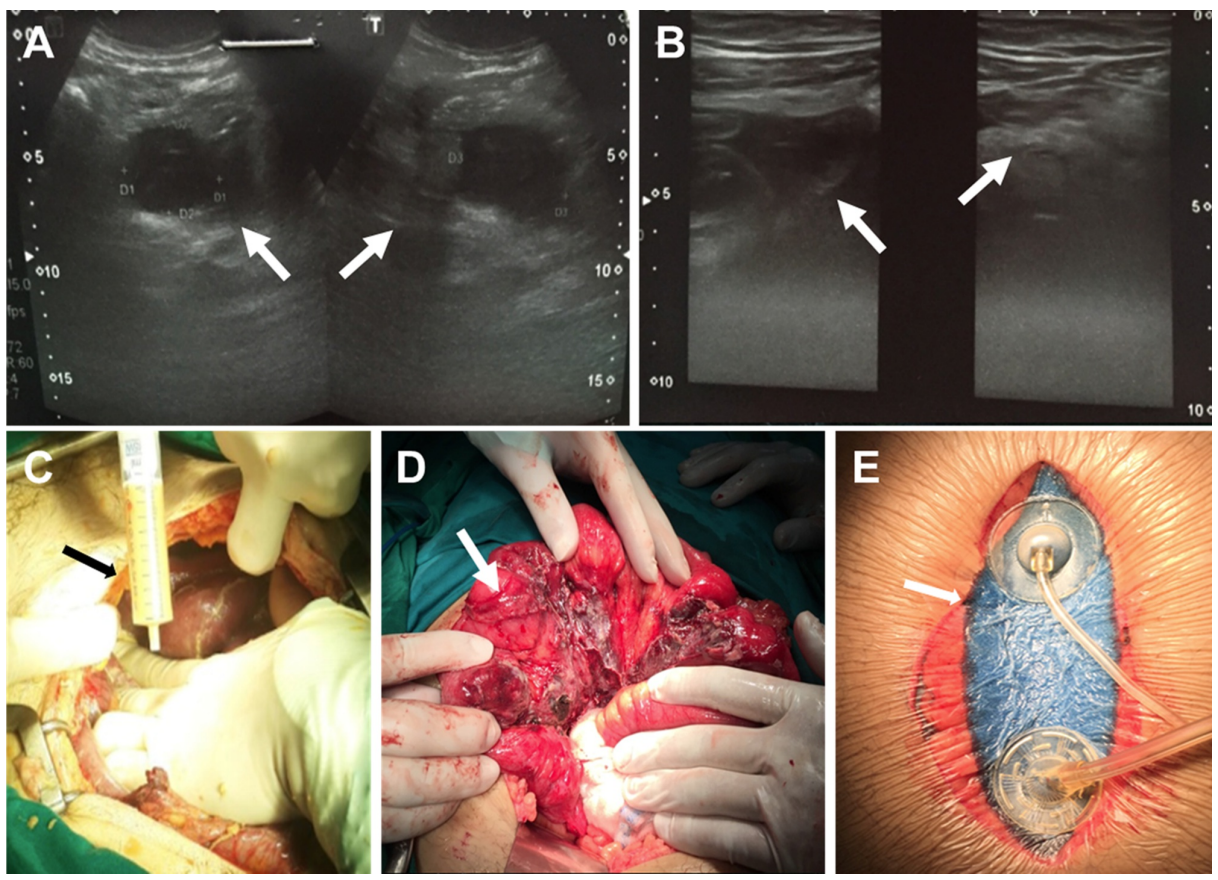

FIGURE 8: Perforated appendicitis with delayed presentation and septic abdomen (Grade 2B)

A. Abdominal ultrasound showing a perforated appendix (view one, arrows); B. Abdominal ultrasound showing a perforated appendix (view two, arrows); C. Abdominal lavage; D. Placement of the ileum (arrow); E. Application of abdominal instillation

The patient underwent exploratory infra-umbilical midline laparotomy, adhesiolysis, aerobic/anaerobic cultures, drainage or debridement of the pelvic/inter-loop abscess, appendectomy, and extensive saline lavage. Intraoperative findings were consistent with perforated appendicitis with retrocecal interloop abscess and mechanical intestinal obstruction and a septic abdomen (OA Grade 2B) (Figures $8 C-8 D$ ). OAI was initiated using $200 \mathrm{cc}$ saline with a 20 -minute dwell time, followed by two hours of continuous negative pressure at $-125 \mathrm{mmHg}$. Intravenous antibiotics were given. After three days, the patient was taken to the OR for abdominal washout. During the second operation, no residual purulent fluid or fibrin deposition was observed and minimal adhesions required some visceral displacement. The persistence of some fluid warranted a prolonged OAI approach for a second 72-hour term (Figure $8 E$ ). Three days after the reexploration surgery, the patient returned to the OR for surgical abdominal washout, definitive closure, and drain placement. Seven days after primary closure, the patient was discharged from the hospital in good condition.

\section{Discussion}

Historically, surgeons were trained to perform a definitive operation for a patient with severe abdominal trauma, sepsis, hemorrhage, or other conditions, as an open abdomen following laparotomy was considered sub-optimal treatment. However, the closure of the abdomen sometimes occurred too quickly, which often led to complications and even death $[3,14]$. This initial multicenter, pooled case series serves as a first look into the applicability of combining the TAC technique using NPWT with OA instillation performed by different surgeons in a variety of clinical settings.

The addition of fluid instillation to traditional OA NPWT has been previously reported as a valid method of managing abdominal sepsis in patients requiring an OA approach [11,15-18]. D'Hondt et al. reported on the instillation of an antibiotic solution following pancreatic surgery using OAI, concluding that OAI was a promising adjunctive treatment when traditional therapy fails to manage the infection [15]. A 12-patient pilot study by Jimenez-Fuerteset al. and a successful case by Nisi et al. showed similar results in patients with abdominal sepsis or secondary septic peritonitis with OAI using saline [16-17]. In the largest case series to date, Sibaja et al. reported higher fascial closure rates, displayed lower mortality rates, and reduced hospital and ICU length of stay without complications resulting from OA instillation following the use of OAI in 48 patients with severe abdominal sepsis [19]. In our study, the use of OAI with normal saline, bacitracin, or hypochlorous acid solutions, in addition to damage control surgery, allowed for abdominal closure without complications. 
review assessed inadvertent perioperative hypothermia (a drop in core temperature to below $36^{\circ} \mathrm{C}$ ) $[20]$. According to this review, there appears to be a link between the instillation of fluid into the abdominal cavity and hypothermia. The investigators reported no statistically significant differences in core body temperature or shivering between individuals given warmed and room temperature irrigation fluids [20]. In our study, no evidence of hypothermia as a meaningful clinical complication was found with a low (5 L) or high volume $(25 \mathrm{~L})$ of direct abdominal irrigation. However, it is important that clinicians closely observe their patients during fluid instillation to ascertain the presence of reduced body temperature as a potential complication of the OA and intraabdominal fluid instillation.

Many types of instillation fluids have been utilized in the abdominal cavity with different results. Antibiotics have been regularly used as part of the instillation of the abdomen [21]. While many studies have been conducted with regards to their use in this context, the benefits of adding antibiotic solutions to the septic abdominal cavity remain unclear, and its pharmacological-clinical effectiveness remains unknown [22]. Other types of solutions such as anesthetics, more commonly, bupivacaine, have been utilized as postsurgical analgesia with varying levels of success [23-24]. Hypochlorous acid is also utilized as an instillation fluid to reduce abdominal fluid viscosity, thus increasing the removal of inflammatory ascites and septic material in the abdominal cavity [25]. There is little evidence of which instillation fluid is superior. While there are studies in which the use of antibiotics in the instilled solutions appears to yield better results, other studies fail to show the added benefit. The same case can be made for the use of anesthetics, which also fails to provide consistently superior pain management.

\section{Conclusions}

Recent therapeutic advances in the management of OA have improved patient mortality and morbidity. This case series suggests that the OA NPWT technique is an effective alternative to repeated multiple laparotomies in patients with diffuse abdominal sepsis. The addition of saline solution or hypochlorous acid solutions to OA NPWT in a programmed, controlled manner may offer the clinician an effective adjunctive therapy for the treatment of the complex septic abdomen. The use of OAI in these seven patients resulted in abdominal closure while minimizing septic complication in all patients. However, future studies are needed to fully assess the clinical benefit of OAI.

\section{Additional Information \\ Disclosures}

Human subjects: Consent was obtained by all participants in this study. CHRISTUS TRINITY MOTHER FRANCES HOSPITAL IRB issued approval NONE REQUIRED. This study consists of 7 patients from 3 different institutions. This represents a pooled case study. No single institution had more than 3 patients. All patients signed informed HIPPA approved consents, including the use of photographic data. All authors removed HIPAA identifiers (including unique patient characteristics) from the data prior to submission and possible publication of this article (and as per HIPPA guidelines, did not need to obtain signed privacy authorization, even though HIPPA compliant consent was also obtained). By HIPPA definition, a case report is an activity to develop information to be shared for medical/educational purposes. All the authors asked and receive guidance from their respective IRB boards; as no single institution had more than 3 cases, no review was required or recommended. Animal subjects: All authors have confirmed that this study did not involve animal subjects or tissue. Conflicts of interest: In compliance with the ICMJE uniform disclosure form, all authors declare the following: Payment/services info: LG Fernández, PJ Sibaja, MJ Kaplan, and MR Matthews are consultants for KCI, an ACELITY Company. AA Sanchez-Betancourt has no conflict of interest to declare. Financial relationships: All authors have declared that they have no financial relationships at present or within the previous three years with any organizations that might have an interest in the submitted work. Other relationships: All authors have declared that there are no other relationships or activities that could appear to have influenced the submitted work.

\section{References}

1. Miller RS, Morris JA Jr, Diaz JJ Jr, Herring MB, May AK: Complications after 344 damage-control open celiotomies. J Trauma. 2005, 59:1365-1374. 10.1097/01.ta.0000196004.49422.af

2. Sutton E, Bochicchio GV, Bochicchio K, Rodriguez ED, Henry S, Joshi M, Scalea TM: Long term impact of damage control surgery: a preliminary prospective study. J Trauma. 2006, 61:831-836. 10.1097/01.ta.0000239504.35149.c5

3. Brenner M, Bochicchio G, Bochicchio K, et al.: Long-term impact of damage control surgery: a prospective study. Arch Surg. 2011, 146:395-399. 10.1001/archsurg.2010.284

4. Cirocchi R, Birindelli A, Biffi WL, et al.: What is the effectiveness of the negative pressure wound therapy (NPWT) in patients treated with open abdomen technique, a systematic review and meta-analysis. J Trauma Acute Care Surg. 2016, 81:575-584. 10.1097/TA.0000000000001126

5. Batacchi S, Matano S, Nella A, et al.: Vacuum-assisted closure device enhances recovery of critically ill patients following emergency surgical procedures. Crit Care. 2009, 13:R194. 10.1186/cc8193

6. Coccolini F, Biffi W, Catena F, et al.: The open abdomen, indications, management and definitive closure . World J Emerg Surg. 2015, 10:32. 10.1186/s13017-015-0026-5

7. Bjorck M, Kirkpatrick AW, Cheatham M, Kaplan M, Leppaniemi A, De Waele JJ: Amended classification of the open abdomen. Scand J Surg. 2016, 105:5-10. 10.1177/1457496916631853 
8. Tinkoff G, Esposito TJ, Reed J, Kilgo P, Fildes J, Pasquale M, Meredith JW: American association for the surgery of trauma organ injury scale I: spleen, liver, and kidney, validation based on the National Trauma Data Bank. J Am Coll Surg. 2008, 207:646-655. 10.1016/j.jamcollsurg.2008.06.342

9. Hinchey EJ, Schaal PG, Richards GK: Treatment of perforated diverticular disease of the colon . Adv Surg. 1978, 12:85-109.

10. Rotondo MF, Schwab CW, McGonigal MD, et al.: 'Damage control': an approach for improved survival in exsanguinating penetrating abdominal injury. J Trauma. 1993, 35:375-383.

11. Matthews MR, Quan AN, Shah AR, et al.: Hypochlorous acid for septic abdominal processes using a unique negative pressure wound therapy system: a pilot study. Surg Science. 2018, 9:412-421.

10.4236/ss.2018.911048

12. Linder MM, Wacha H, Feldmann U, Wesch G, Streifensand RA, Gundlach E: The Mannheim peritonitis index. an instrument for the intraoperative prognosis of peritonitis [Article in German]. Chirurg. 1987, 58:84-92.

13. Turley RS, Barbas AS, Lidsky ME, Mantyh CR, Migaly J, Scarborough JE: Laparoscopic versus open Hartmann procedure for the emergency treatment of diverticulitis: a propensity-matched analysis. Dis Colon Rectum. 2013, 56:72-82. 10.1097/DCR.0b013e3182749cf5

14. Vargo D, Richardson JD, Campbell A, et al.: Management of the open abdomen: from initial operation to definitive closure. Am Surg. 2009, 75:S1-S22.

15. D'Hondt M, D'Haeninck A, Dedrye L, Penninckx F, Aerts R: Can vacuum-assisted closure and instillation therapy (VAC-instill therapy) play a role in the treatment of the infected open abdomen. Tech Coloproctol. 2011, 15:75-77. 10.1007/s10151-010-0662-4

16. Jimenez-Fuertes M, Ruiz-Tovar J, Duran-Poveda M, Garcia-Olmo D: Negative pressure therapy with intraperitoneal saline instillation in the open septic abdomen. Int J Surg Res. 2016, S3:1-4.

17. Nisi F, Marturano F, Natali E, Galzerano A, Ricci P, Peduto VA: VAC therapy with long term continuous saline infusion for secondary septic peritonitis: a new strategy for the reduction of perioperative risks. Int J Surg Case Rep. 2017, 32:62-65. 10.1016/j.ijscr.2017.02.010

18. Alvarez PS, Betancourt AS, Fernandez LG: Negative pressure wound therapy with instillation in the septic open abdomen utilizing a modified negative pressure therapy system. Ann Med Surg (Lond). 2018, 36:246251. 10.1016/j.amsu.2018.10.007

19. Sibaja P, Sanchez A, Villegas G, Apestegui A, Mora E: Management of the open abdomen using negative pressure wound therapy with instillation in severe abdominal sepsis: a review of 48 cases in hospital Mexico, Costa Rica. Int J Surg Case Rep. 2017, 30:26-30. 10.1016/j.ijscr.2016.11.024

20. Campbell G, Alderson P, Smith AF, Warttig S: Warming of intravenous and irrigation fluids for preventing inadvertent perioperative hypothermia. Cochrane Database Sys Rev. 2015, 13:CD009891. 10.1002/14651858.CD009891

21. Hau T, Ohmann C, Wolmershauser A, Wacha H, Yang Q: Planned relaparotomy vs relaparotomy on demand in the treatment of intra-abdominal infections. Arch Surg. 1995, 130:1193-1197. 10.1001/archsurg.1995.01430110051009

22. Rambo WM: Irrigation of the peritoneal cavity with cephalothin. Am J Surg. 1972, 123:192-195. 10.1016/0002-9610(72)90332-7

23. Sharan R, Singh M, Kataria AP, Jvoti K, Jarewal V, Kadian R: Intraperitoneal instillation of bupivacaine and ropivacaine for postoperative analgesia in laparoscopic cholecystectomy. Anesth Essays Res. 2018, 12:377380. 10.4103/aer.AER_6_18

24. Ali PB, Cotton BR, Williamson KM, Smith G: Intraperitoneal bupivacaine or lidocaine does not provide analgesia after total abdominal hysterectomy. Br J Anaesth. 1998, 80:245-247. 10.1093/bja/80.2.245

25. Matthews MR, Quan AN, Wier AS, Foster KN, Caruso DM: Temporary abdominal closure combined with an irrigating system utilizing hypochlorous acid solution to decrease abdominal mucopurulence. Eplasty. 2018, 18:e12. 